\title{
Exploration of the Antimicrobial Resistance and Their Remedy in Living World: A Brief Study
}

\author{
Md. Ashiqur Rahamn ${ }^{1 *}$, Bushra Rahman ${ }^{2}$, Tanvir Ahammed ${ }^{3}$, and Imdadul Haque Sharif ${ }^{4}$ \\ ${ }^{1}$ Dept. of Pharmacy, ASA University Bangladesh, Dhaka, Bangladesh; ${ }^{2}$ Department of Pharmaceutical Sciences North South \\ University, Bangladesh; ${ }^{3}$ Dept. of Microbiology and Hygiene, Bangladesh Agricultural University, Bangladesh; and ${ }^{4}$ Dept. of \\ Biotechnology and Genetic Engineering, Bangabandhu Sheikh Mujibur Rahman Science \& Technology University, \\ Gopalganj, Bangladesh.
}

*Correspondence: 7upmilon@gmail.com

\begin{abstract}
Antimicrobial resistance (AMR) alarms the effective control and treatment of growing infections of bacterial, parasitic, viral and fungal infections. This report, for the initial stage, examines the current state of monitoring and information regarding AMR at the global country level, particularly antibacterial resistance (ABR). Main research and public health effects of ABR; high rates of resistance to bacteria causing common health-carerelated and community-acquired infections (e.g., urinary tract infections, pneumonia) have been perceived in all WHO areas. There are significant gaps in monitoring and lack of criteria for methods, data splitting and coordination. The key findings from AMR scrutiny in disease-specific programs are as; while multidrugresistant TB is an enlarging concern, it has been reported in most cases blank, compromising control efforts. Artemisinin resistance foci in malaria have been picked out in several countries. Further spread or emergence of artemisinin-resistant strains in other regions could jeopardize recent attains in malaria prevention.
\end{abstract}

Keywords: Exploration, Antimicrobial resistance, Remedy, Living world, TB, Treatment, and Antibacterial.

\section{INTRODUCTION:}

Antibiotics are drugs used to prevent and treat bacterial infections. Antibiotic resistance occurs when bacteria change in response to the use of these drugs (Giedraitienè et al., 2011). Bacteria, not humans or animals, become antibiotic-resistant. These bacteria can contaminate humans and animals, and the infections they cause are harder to treat than diseases caused by non-resistant pathogen. Antibiotic resistance results in higher treatment costs; longer hospital stays, and enlarges mortality (Džidić et al., 2008).

The world urgently needs a way to prescribe and use antibiotics. Even if new drugs are developed without a

UniversePG I www.universepg.com change in behavior, AMR will remain a vital threat. Behavior alters must also include actions to decrease the spread of contaminants through immunization, hand cleaning, practicing safer sex, and fresh food hygiene (WHO, 2020).

1.1. Rational use of the antibiotics - The developpment and spread of AMR is less due to overuse, misuse and indiscriminate use of antimicrobials by physicians, nurses and pharmacists, due to disobedience and self-medication by patients and their use in animal husbandry and agriculture (Rice, 2008). It is estimated that $70-80 \%$ of prescriptions for antimicrobials are probably advised unnecessarily by the health professionals. Although most common colds 
and diarrhea episodes are primarily viral, antimicrobials are used indiscriminately. The causes of over-the-counter medications are often lack of confidence, peer pressure, patient stress, and pharmaceutical agency stress and a key driver of resistance to antimicrobial use. Poverty in antibiotics and useless quit access identified as a major cause of development (Rahman et al., 2019).

Another common cause of developmental impairment is improper diagnosis. In many cases adequately equipped diagnostic labora-tories force physicians to prescribe antibiotics in the vicinity, thus increasing the patient's chances of taking the wrong antibiotics. In addition, the ready availability of over-the-counter and sales promotion projects by pharmaceutical manufacturers also leads to the promotion of the use of Indus-Criminate, thereby increasing the likelihood of developing resistance. Counterfeit drugs also contribute to the development of resistance. This includes incorrect ingredients, or less active ingredients. In some cases, drug toxins can cause disability or even death. Media influence has also played a role in the development of resistance. Patients often demand or view antibiotics for their readings based on the demand for the disease ( $\mathrm{Li}$ and Nikaido, 2004). It is detrimental in terms of increasing the cost of therapy, unnecessary adverse drug reactions, treatment failures, declining quality of care and the worst of these.

The bacterial infections that contribute the most to human death and illness are also the most obvious of the emerging AMR: diarrhea, respiratory infections, meningitis, sexually transmitted diseases, and hospital-acquired infections (Del Pozo and Patel, 2007). Some important 626NFI-2011examples include penicillin-resistant Streptococcus pneumoniae, vancomycin-resistant Enterococci, methicillin-resistant Staphylococcus aureus (Pucci and Dougherty, 2002), multi-resistant Salmonella typhi, Shigella dysenteriae, Neisseria gonorrhea, Pseudomonas aeruginosa (Schweizer, 2003; Sharma et al., 2014) and multiresistant Mycobacterium tuberculosis. The development of resistance to drugs commonly used to treat $P$. falciparum malaria is of particular concern, as is the emerging resistance to antiretroviral drugs.
2. Mechanisms of AMR - Anti-microbial resistance genes may be carried on the bacterial chromosome, plasmid, or transposons. Mechanisms of drug resistance fall into several broad categories, including drug inactivation/alteration, modification of drug binding sites/targets, changes in cell permeability resulting in reduced intracellular drug accumulation, and biofilm formation (Wilson, 2014).

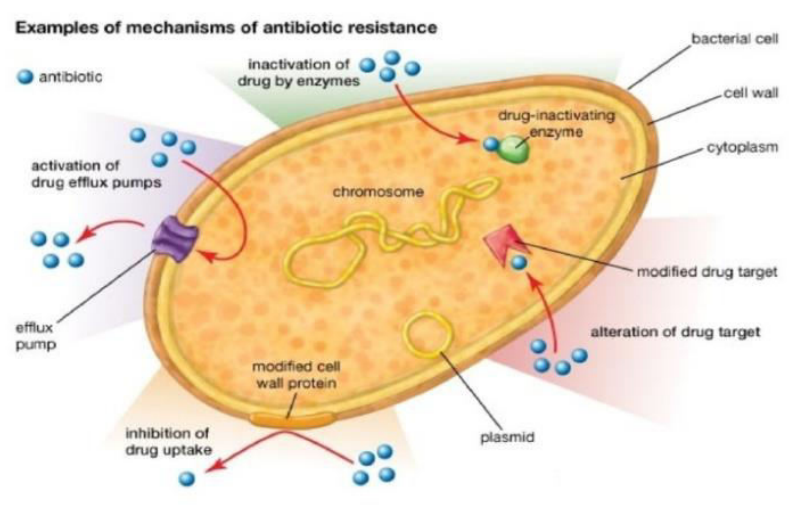

Fig 1: Mechanisms of Antimicrobial resistance.

2.1. Drug inactivation or alteration - Many bacteria produce enzymes that irreversibly modify and inactivate the antibiotics, such as $\beta$-lactamases, aminoglycoside-modifying enzymes, or chloramphenicol acetyltransferases (Bush and Jacoby, 2010). One of the well-characterized enzymes is $\beta$-lactamases. They are highly prevalent and act by hydrolyzing the $\beta$ lactam ring which is present in all $\beta$-lactams; thus, all penicillins, cephalosporins, monobactams, and carbapenems are essential to their activity $\beta$-lacta-mases are classified using two main classification systems: the Ambler scheme and the Bush-Jacoby-Medeiros system, which classifies the most clinically important $\beta$-lactamases as those produced by Gram-negative bacteria Ambler class A enzymes consist of penicillinase, cephalosporinase, broad-spectrum $\beta$-lactamases, extended-spectrum $\beta$-lactamases (ESBLs), and carbapenemases (Jacoby and Munoz-Price, 2005; Bonnet, 2004). They can inactivate penicillins (except temocillin), third-generation oxyimino-cephalosporins (e.g., cefta-zidime, cefotaxime, and ceftriaxone), aztreonam, cefamandole, cefoperazone, and methoxycephalosporins. Class A enzymes can also be inhibited by $\beta$-lactamase inhibitors, such as clavulanic acid, sulbactam, or tazobactam. 
The Emblar Class A group contains several notable enzymes, including ESBLs (mainly TM, SHV, and CTX-M type) and KPC. Tem-type enzymes were first identified in E. coli from Greek patients in 1965, after a patient named Temonira (Laverty et al., 2014). Tem1 is not only among the bacteria of the enterobacteriaceae family, it is also widespread in non-fermenting bacteria such as Aruginosa. Currently, TEM enzymes are among the most common group sulfidril variable (SHV) enzymes in E. coli, SHV-1 being the most clinically relevant and the most common representing $K$. pneumonia. The coding genes of TM and SHV enzymes have the highest mutation rates, resulting in a high level of diversity of enzyme types and thus increased antibiotic resistance. New Timonia, Baumani (Høiby et al., 2010) and CTX-MS have been detected in Escap germs including Aruginosa and Enterobacter species (Rice, 2010). Some of the highest prevalence and significant clinical impact are associated with the extended-spectrum $\beta$-lactamases in $K$. pneumonia. Carbapenemases are also prevalent in clinical bacterial isolates such as $K$. pneumonia such as KPC-1 that results in resistance to imipenem, meropenem, amoxicillin, piperacillin, ceftazidime, aztreonam, and ceftriaxone.

Ambler class B enzymes, or group 3 enzymes as classified by the Bush-Jacoby system include metallo$\beta$-lactamases (MBLs), which require $\mathrm{Zn}^{2+}$ as a cofactor. Bacteria that produce these enzymes show resistance to all $\beta$-lactams, including penicillins, cephalosporins, carbapenems, and $\beta$-lactamase inhibitors, except aztreonam (Yong et al., 2009). Genes encoding MBLs are found in plasmids; therefore, they are easily transmitted to other microorganisms. The most common metallo- $\beta$-lactamases (MBLs) are imipenemase metallo- $\beta$-lactamases (IMP), Verona integron encoded metallo- $\beta$-lactamases (VIM), and the newly described New Delhi metallo-beta-lactamase-1 (NDM-1) enzymes. IMP-type MBLs have generally been found in $P$. aeruginosa, $K$. pneumoniae, $A$. baumannii, and Enterobacter cloacae, while VIMtype enzymes have been extracted mostly in $P$. aeruginosa and A. baumannii. NDM-1-type enzymes have been extracted from $K$. pneumoniae and $E$. cloacae. The Ambler class $\mathrm{C}$ group consists of several important enzymes, including penicillinase and cephalosporinase, such as AmpC $\beta$-lactamase, which results in low level resistance to narrow-spectrum cephalosporin drugs.

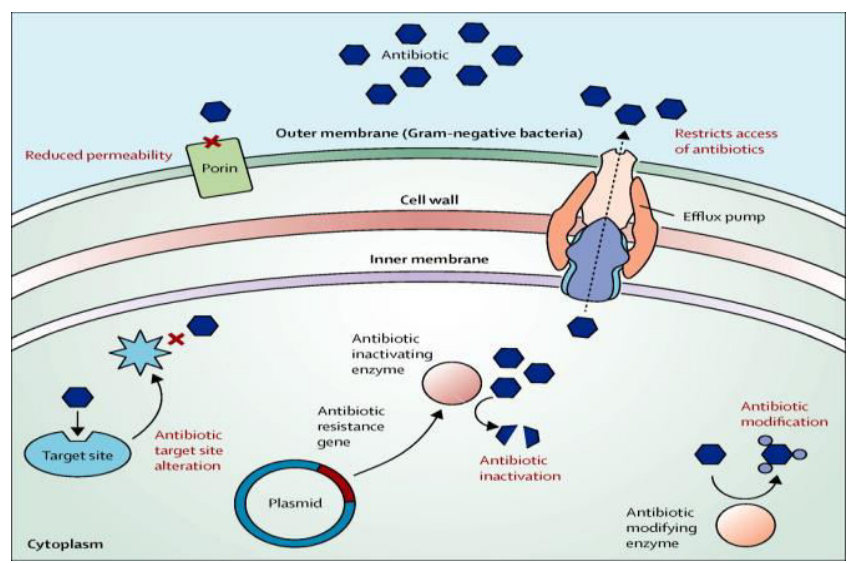

Fig 2: Drug inactivation or alteration process.

Chromosomally encoded ampicillin is usually Bacteria in the P. aruginosae and Enterobacteriaceae family are detected in such Enterobacter species where their production is usually very low and does not limit any clinically relevant resistance but may be uncontrollable during drug therapy. Nevertheless, the acquisition of transmissible plasmids from other bacteria can lead to the overproduction of AmpC $\beta$ lactamase in some organisms ordinarily lacking the gene encoding for chromosomal AmpC, for example, $K$. pneumonia. The AmpC $\beta$-lactamases inactivate aztreonam, all penicillin, and most cephalosporins and are not susceptible to inhibition by most $\beta$-lactamases inhibitors except avibactam, a new non- $\beta$-lactam $\beta$ lactamase inhibitor antibiotic (Jacoby, 2009). Ambilar class D contains types of enzymes such as oxacillin hydrolyzing enzyme (OXA) and the most popular members of this group, such as OXA-11, and OXA14. OXA enzymes are identified as group 2D following the Bush-Jackbucky scheme, and almost all of these enzymes, except OXA-18, are resistant to $\beta$ lactamase inhibitors. In addition, OXA-type carbapenimes are commonly found in the Actinobacter Spp. At specified Baumani carbapinem hydrolyzing OXA enzymes, which have low catalytic efficiency, together with purine removal and other antibiotic resistance mechanisms, may cause high resistance to carbapenems (Babic et al., 2006; Kumarasamy et al., 2010).

2.2. Modification of the drug binding sites - Some resistant bacteria modify their targets and avoid 
recognition by antimicrobial agents. Gene encrypting for penicillin-binding proteins (PBPs), which enzymes typically anchor to the cytoplasmic layer of the bacterial cell wall and act on assembly and control at a later stage of cell wall building, resulting in expression and the expression of a unique PBP2a is the most influential PBP in MRSA cells compared to native PBPs (PBP1-4). PBP2a has low affinity for all $\beta$ lactam antibiotics and acts as a substitute for the other PBPs, thus enabling the survival of $S$. aureus in the presence of high concentrations of $\beta$-lactam drugs including methicillin acting on cell wall biosynthesis. Bacterial cell wall synthesis in methicillin-resistant Gram-positive organisms can be inhibited by glycolpeptide, which targets acyl-de-alanil-de-alanine (acyl de-ala-de-ala) of peptidoglycan precursors. However, the pepeidoglycan cross-link target encoded by a complex gene cluster (van-a, van-b) changes (de-alade-ala to de-ala-de-lac or de-ala-de-ser), Van-D, VanC, Van-E, and Van-G), E. facium and E. faecalis may increase the resistance to glycoproteins in current clinical use (vancomycin and teicoplanin).

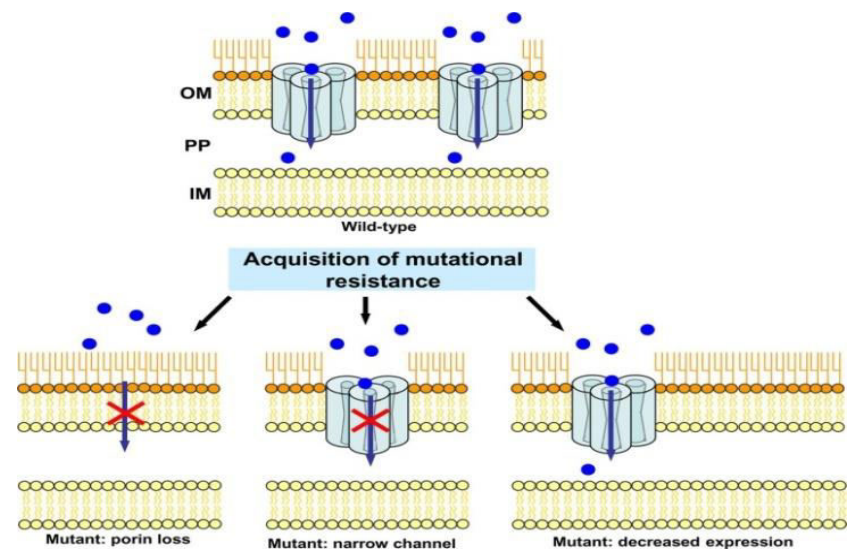

Fig 3: Reduced intracellular drug accumulation.

2.3. Reduced intracellular drug accumulation - The balance of antibiotic intake and elimination minimizes the susceptibility of bacteria to a particular drug. Thus, reducing the amount of antibiotics is able to cross by the bacterial cell membrane, a technique used by pathogen to develop antibiotic resistance (Thomson and Bonomo, 2005). Methods by which bacteria acquire this include the presence of reduced protein channels in the outer membrane of the bacterium to reduce drug penetration and the presence of flow pumps to reduce the amount of drug accumulated in the cell (Vaez et al., 2014).

UniversePG I www.universepg.com
2.3.1. Porin loss - The outer membrane of Gramnegative bacteria contains a protein called porin that forms channels that pass through many hydrophilic substances, including antibiotics (Zhao and $\mathrm{Hu}, 2013$ ). Reducing the amount of $P$. aruginosa porin protein OPRD decreases the tendency of the drug in the cell, allowing the bacteria to develop resistance to immunemodulators. Decreased 29-kDa outer membrane protein (OMP) in other Gram-negative bacteria, such as A. baumania, allows them to be sensitive to imipenem and meropenem drugs (Happy et al., 2018). Multiple-drug resistant $K$. pneumoniae strains also exhibit resis-tant/reduced susceptibility to $\beta$-lactams (such as cephalosporins and carbapenems) by the loss of outer membrane proteins known as OmpK35 and OmpK36 together with the production of resistance enzymes, including AmpC $\beta$-lactamase and newgeneration carbapenemase A, KPC.

2.3.2. Efflux pumps - To enhance antibiotic removal from the intracellular bogie, some bacteria contain membrane proteins that act as exporters for some antimicrobial agents called efflux pumps. These pumps expel the drug from the cells at a high rate, which means the concentration of the drug is never sufficient to avoid antibacterial effects. Most efflux pumps are multidrug transporters that efficiently pump a wide range of antibiotics, contributing to multdrug resistance (Nikaido and Pagès, 2012). Up to date, there are five super families of efflux pumps that have been described. These include the ATP-binding cassette $(\mathrm{ABC})$ group, the tiny multidrug resistance family, the key facilitator super family, the resistancenodulation-division (RND) family, and the multidrug and toxic compound extrusion family.

The most common type of efflux pump in Gramnegative bacteria is the poly selective efflux pump, belonging to the RND super family, which plays a key role in multidrug resistance (MDR) bacterial phenoltypes. This type of pump releases a variety of antibiotics and structurally unrelated molecules such as pigments and bile salts, but also detergents and biocides that are frequently used in medical practice. Acrab-Tolsi and Meksab-OPRM Multidrude efflux pumps usually belong to the RND super family. These are usually chromosomally encoded (Sun et al., 2014). These two flow pumps are necessary for the bacteria 
to survive, especially in the presence of toxic agents. $P$. aruginosa has abundant flow pumps, of which four powerful RND-type multidrug resistance efflux pumps (MAX) are capable of eliminating toxic compounds from the periplasm and cytoplasm. Two of these efflux pumps, MexAB-OprM and MexCD-OprJ, are responsible for resistance to at least three main classes of antibiotics, namely, carbapenems, fluoroquinolones, and amino-glycosides. Studies have shown that Over-expression of maxXY-ORPM from $P$. eruginosa results in resistance to aminoglycosides, fluoroquinolones, and specific antipizodomonal cephalosporins. More- many clinical $P$. aruginosa also isolated MaxCD-ORPJ and MaxF-ORPN. Enterobacter aerogens and $K$. Pneumonia clinical isolates have been shown to increase the tendency to over-produce these flow pumps.

The over-expression of the crab flow pump, together with the reduced expression of the pores, is characteristic of the impenem-resistant e-erosion MDR strains. In these bacteria, efflux Pump also releases other irrelevant antibiotics such as fluoroquinolones, tetracycline and chloramphenicol. $A$. baumani isolates may also exhibit an MDR phenotype by the presence and excessive expression of the RND fuel pump AdeABC. This pump is attached with resistance to a large range of antibiotics, including fluoroquinolones, $\beta$-lactams, tetracyclines macrolides/ lincosamides, chloram-phenicol, and aminoglycosides. Like $P$. aeruginosa, A. baumannii porins also show very low permeability. RND-type efflux pumps include AdeABC, AdeDE, Ade-FGH, and AdeIJK aminoglycosides, fluoro-quinolones, erythromycin, tetracycline, and chloramphenicol. Finally, the synergistic carry off the external membrane barrier of Maltdrew influx pumps is important for the resistance of many elements. For example, the key porin visualized by $P$. aeruginosa is $\mathrm{OprF}$, which has much lower permeability than E. coli OmpF, making the efflux pump activity more active in resistant genera.

2.4. Biofilm formation - Biofilms are complex microbial communities living as a thin layer on biotic or abiotic surfaces, implanted in a matrix of extracellular polymeric substances created by the biofilms themselves (Uddin et al., 2017). The microorganisms in the biofilm can interact with each other as well as with the UniversePG I www.universepg.com environment. The main components of the matrix are extracellular polymeric substances secreted, consisting mainly of polysaccharides, proteins, lipids, and extracellular DNA from microorganisms. There are three key steps for biofilm formation. The first step is adhesion, which occurs as cells reach a surface and anchor to the site. The second step is growth and maturation, which happens as the microbes begin to generate the exopolysaccharide that establishes the matrix and then mature from micro colonies to multilayered cell bunches. The final step is isolation, which can be divided into two types: active and passive. Active isolation is initiated by bacteria, for example, through corium sensing and enzymatic degradation of the biofilm matrix (Wright, 2005). In contrast, passive isolation is caused by external forces, such as liquid shear, scraping, and human intervention. It may be argued that the main causes of AMR are not classical drug resistance, afflux pumps, target site modification or enzymatic degradation (Alam et al., 2017). It is likely that the matrix of biofilms provides a mechanical and biochemical shield that provides the conditions needed to attenuate the activity of the drugs. Under these situations it is very hard to eliminate pathogen using traditional antibiotics. Besides, when the bacteria learn nutrient scarcity, they could become tolerant to antibiotics. This may explain the seemingly greater antibiotic resistance of cells in the deeper layers of a biofilm. The most common pathogen in healthcare setting biofilms is $S$. aureus, $P$. aruginosa, A. baumni and $K$. pneumonia (Magill et al., 2014; and Klevens et al., 2007).

3. Effect of AMR - The impact of AMR can be accessed from the perspective of the hospital, a thirdparty payer, the patient, and society. Studies that examine one perspective can underestimate the full carry off AMR; therefore, it is important to recognize the perspective of a study to appropriately interpret its results.

3.1. Hospital perspective - Hospital perspectives on the effects of prevention have been studied frequently. It is relatively easy to retrieve information on hospital sickness, mortality, and costs related to AMR, and hospitals are more likely to implement changes in response to hospital-level evaluated data (Islam et al., 2020). 


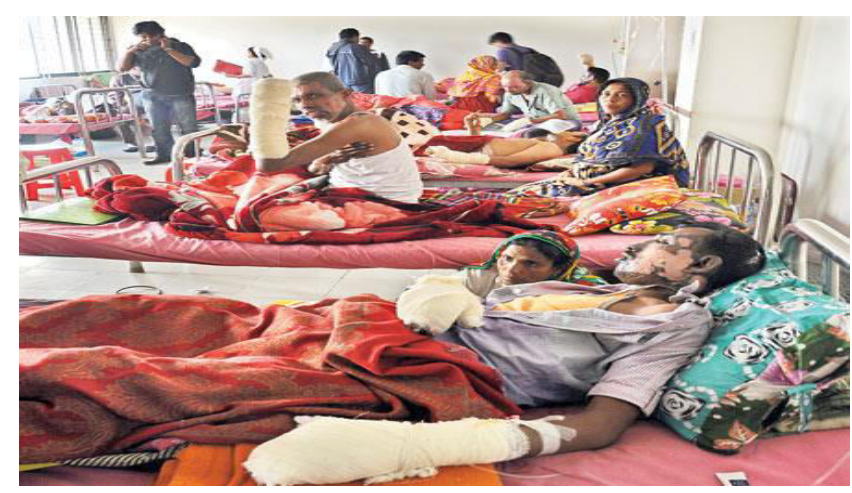

Fig 4: Effect of Antimicrobial resistance.

Several recently published studies have evaluated the effects of antibiotic resistance by assessing hospital admissions mortality and hospital admissions length. Few studies have examined economic results. It is important to recognize that the hospital's view of the effects of AMR provides a limited view of the healthcare impact of prevention because significant portions of clinical care are now provided in rehabilitation facilities, nursing homes and at home. These sites contain limited information about costs because thirdparty providers make it difficult to combine claims data with the results of microbiology without compromising the privacy of the information they collect. Antibiotics resistance can easily spread through hospital. Random people stay in hospital and they may have different type of resistance. As a result superbug may develop. A new born baby or a normal people who come to see patient can be affected.

3.2. Patient Perspective - Measurements of mortality and long of hospitalization assess the short-term direct impact of resuscitation of the affected patient. However, resistant infections can have significant effects on the indirect and long-term consequences. For example, a patient with a history of MRSA infection who presents with a new fever is usually placed in isolation and empirically treated with vancomycin, even though he or she may not have MRSA infection (Ekhlas et al., 2014). Other patientoutcomes at the required level of further utterance include the long-term effects of future health-resistant infections, extended hospital admissions and work related to subsequent recovery, and the loss of family time and even the emotional effects of resistant infections. The effects of AMR also occur in patients who have not been infected with the resistant UniversePG I www.universepg.com organism. Due to the increasing rate of resistance among common pathogens, broad-spectrum agents are now needed for emetic therapy of many common bacterial infections. These agents are usually more expensive, have a more detrimental effect on the protective microflora, and are sometimes more toxic or less effective. For example, third-generation cephalosporins or fluoroquinolones are recommended for the treatment of hospitalized patients with communityacquired pneumonia, while resistance rates go up to a certain level of damage to narrow-spectrum agents such as penicillin for the treatment of common diseases.

3.3. Societal perspective - Current perceptions about the effects of AMR in society as a whole are limited. The Office of Technology Assess-Man estimates that the national cost of antibiotic resistance in the United States is \$4 billion per year at; 1995; However, this assessment only took into account directly affected patients and not other provisions of prevention, which would probably multiply the prognosis. Further study of the impact of resistance beyond the patient and hospital levels will be essential to guide decisionmakers (Carmeli et al., 1999; Carmeli et al., 2002; and US Congress, 1995).

\section{Source of antimicrobial resistance}

\subsection{Mechanism of transferring antimicrobial resis-} tance - Genetic material can be transferred between bacteria by several means, most often by: Conjugation, Transformation, and Transduction.

4.1.1 Conjugation - Conjugation is handled by a specific kind of round DNA called a plasmid, which replicates freedom of the chromosome. Many plasmids bear genes that consult resistance to antimicrobials.

4.1.2. Transformation - During this process, genes are transferred from one bacterium to another as "naked" DNA. When cells die and separate, DNA can be released into the surrounding environment. Other nearby bacteria can transmit this free DNA and incorporate it into their own DNA. This DNA may contain advantageous genes, such as antimicrobial resistant genes and benefit the recipient cell. 


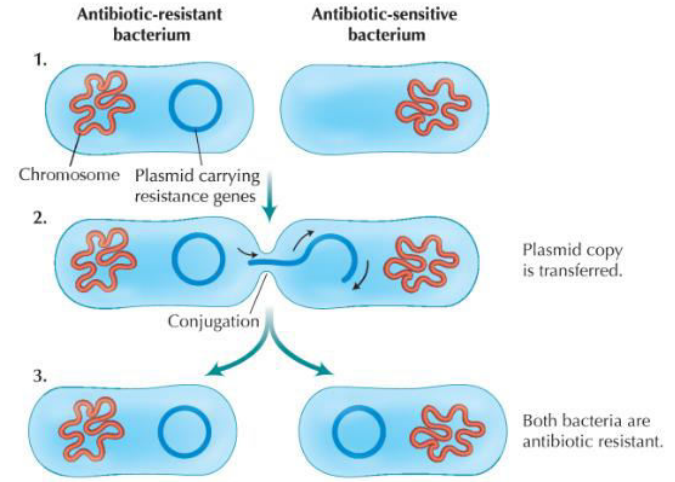

Fig 5: Conjugation process.

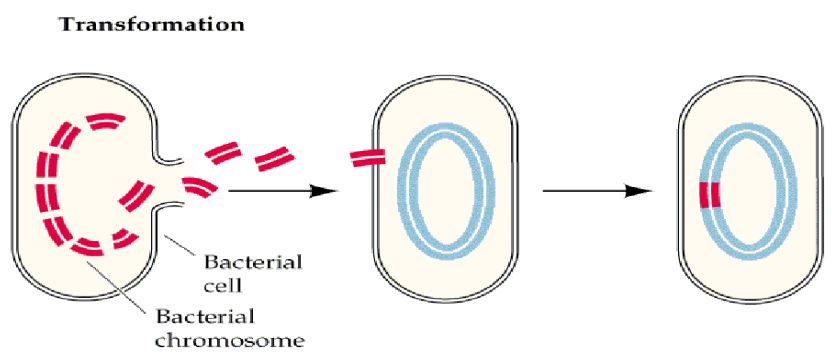

Fig 6: Transformation process.

4.1.3. Transduction - In this process, the bacterial DNA is transferred from one bacterium to another within the virus transmitted from one bacterium to another. These viruses are called bacteriophages or phages. When a phase infects a bacterium, it basically takes the genetic processes of the bacteria to create more phases. During this process, bacterial DNA may inadvertently attach to new phase DNA. After bacterial death and lysis, this new phase infects other bacteria. This brings along genes from the previously infected bacterium (US FDA, 2004).

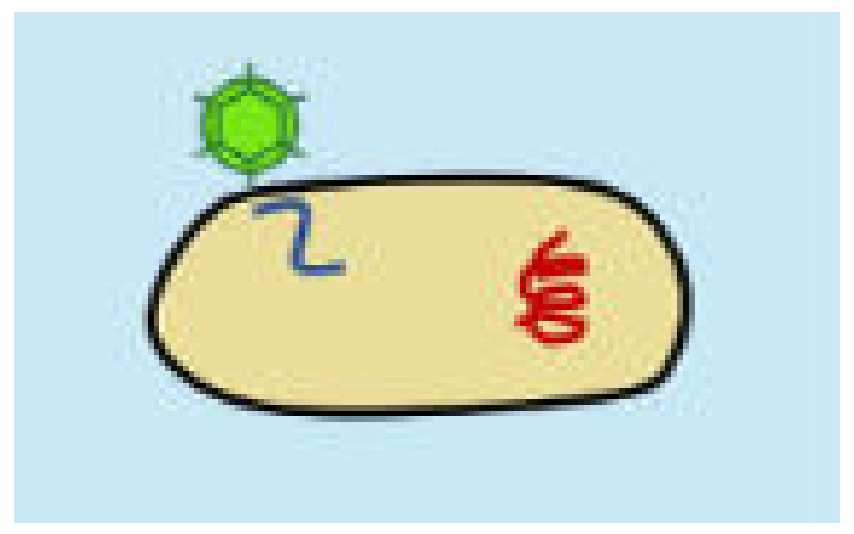

Fig 7: Transduction process.
4.2. Pathways of spreading AMR - Due to the infectious nature of resistant bacteria, a populationlevel framework is needed. Therefore, we are expanding a population prevalence model that accounts for host-to-host transmission with a multimedia environmental model of antibiotic resistant genes (Mondol et al., 2018). The combined model, shown below, will be used to examine the flows of antibiotic resistance between different environmental compartments and the carry off these flows on the risk of exposure from the environment. The environmental model will describe the evolution, dissemination, and persistence of antibiotic resistant genes in soil, surface water, and air. Spatial heterogeneity is included by the addition of physical inputs that affect the growth of bacterial populations or the emergence of resistance: the presence of metals, and antibiotics, biofilms, temperature, nutrition levels, and saturation. The flow of these inputs and the genes of resistance from humans and animals will be parameterized depend on experimental finding. We will use data published in Enterobacteria to parameterize and validate environmental multi-media models. Antobacteria are necessary for their role as human pathogens and contain significant information about their presence in the environment. To understand the interactions between different sections of environmental models, we will adopt diagnostic tools that are used for toxic multimedia models. Recent microbial exposure assessment approaches will be utilized to estimate human exposure to environmental resistance.

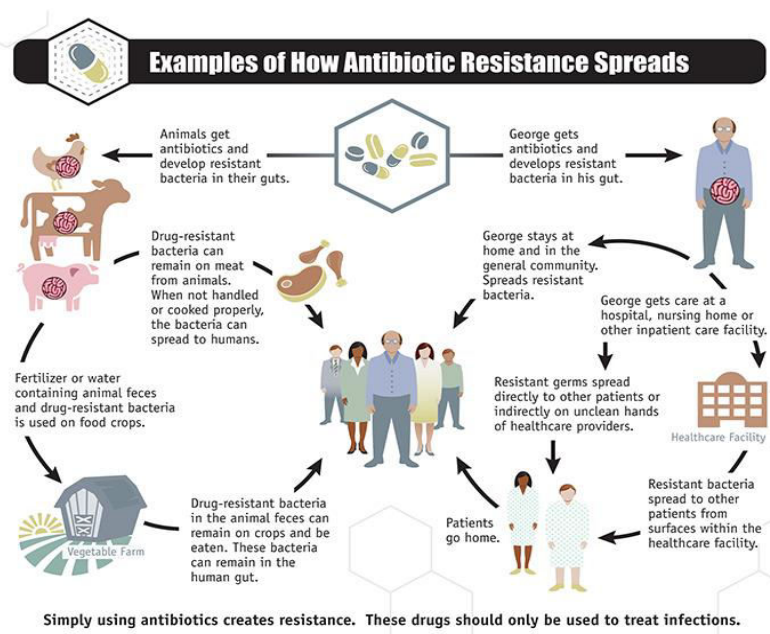

Fig 8: Pathways of spreading AMR. 
To execute a sensitivity test assessing the most vital factors driving risk and remaining un-knowns we will use a Regional Sensitivity approach (Olivier Jolliet, 2005).

5. Most antibiotics resistance - Based on the urgency of the need for new antibiotics, the WHO list is divided into three categories: critical, high and medium priority. Among the most critically acclaimed groups are the multi-drug drug-resistant bacteria that pose a particular threat to hospitals, nursing homes, and especially to patients who need tools such as ventilators and blood catheters. These include Achinetobacter, Pseudomonas and various Entero-bacteria (together with Klebsiella, E. coli, Serratia, and Proteus). These can cause serious and often fatal infections, such as bloodstream infections and pneumonia. These bacteria have become increasingly resistant to antibiotics, with carbapenems and thirdgeneration cephalosporins being among the best available antibiotics for the treatment of multi-drug drug-resistant bacteria. The second and third levels of the list contain other growing drug-resistant bacteria in the upper and middle priority categories that cause gonorrhea and common foodborne illnesses caused by Salmonella. Tuberculosis- whose resistance to conventional control has been growing in recent years- was not included in the list because it is targeted by other, dedicated programmes. Other bacteria that were not included, such as Streptococcus A and B and Chlamydia, have a lower level of resistance to existing treatments and are not currently a threat to public health.

\subsection{List of Categories}

\subsection{Priority 1: Critical}

- Acinetobacter baumannii, carbapenem-resistant

- Pseudomonas aeruginosa, carbapenem-resistant

- Enterobacteriaceae, carbapenem resistant, ESBL- producing

5.2. Superbug - The term Superbug is an acronym used to describe a microorganism that is resistant to at least one or more commonly used antibiotics (Shahen et al., 2019). Emerging superbugs may include multiple drug-resistant Clostridium difficile, VRSA (vancomycin-resistant $S$. aureus), and NDM E. coli (New Delhi's metallic-beta-lactamase-resistant E. coli) and UniversePG I www.universepg.com some strains of gonorrhea. In 2013, the CDC established a superbug site as a "threat" to 18 genres and species due to AMR. These are categorized according to their potential as the cause of urgent, serious and serious health problems; MRSA is ranked as critical (WHO, 2017).

\section{Current situation of antibiotic resistance in} Bangladesh - Bangladesh is one such a country where there is a severe lack of local information on AMR. A number of studies hosted at different institute aim to generate knowledge on AMR considering it as a one Health problem. Antibiotic resistance is one of the biggest threats to global health. It can affect anyone, of any age, in any country. Although antibiotic resistance occurs naturally, overuse and misuse of antibiotics in humans and animals is accelerating the process. Steps can be taken at all levels of society to reduce the impact and limit the spread of resistance, including the public, who can help by preventing infection through good hygiene and vaccination, only using antibiotics when prescribed by a certified health professional, taking the full course, and never sharing or using left-over antibiotics. For this reason, it is critical that people understand the problem, and the way in which they can change their behaviour. In relation to this, the results of this survey show there is much to be done. They show that although people recognize the problem, they do not fully understand what causes it, or what they can do about it.

\subsection{Transmission dynamics of multi drug resistant} $E$. coli causing urinary tract infection in Bangladesh - Bangladesh appears to be an important reservoir of many multidrug-resistant Enterobacteriaceae strains that are observed globally (Tang et al., 2014). A large proportion of community-acquired urinary tract infections (UTIs) in most parts of the world are caused by a limited number of multidrug-resistant $\mathrm{E}$. coli strains of related offspring that herbalize drugresistant genes shared by disease-biological and environmental villages. Infected $E$. coli (UPEC) strains in some MDR ureters share the same genetic lineage with individuals isolated from the food source, indicating the possibility of food-borne infections of antibiotic-resistant UPEC. The project will examine MDR UPEC's trans-mission dynamics in communities in Dhaka, Bangladesh, to examine how UPEC clonal 
lineages and their drug-resistance determinants spread across a human community, as opposed to clinical settings. This project is designed to determine the quantity, quantity of food and food-producing animals as a reservoir of UPC in a common communityacquired infection-UTI. This will contribute to the formation of more focused public health interventions to prevent the spread of such infections.

\subsection{Spatial and temporal dynamics of antimicro-} bial resistance transmission from the outdoor environment to humans in Bangladesh - The environment is recognized as an important reservoir of antibiotic resistant bacteria, with anthropogenic activities such as agriculture, antibiotic residues and wastewater disposal enhancing the horizontal transfer of antibiotic resistance genes. The recent spread of New Delhi metallo- $\beta$-lactamase-1 (NDM-1) resistance genes through community and environmental pathways in South Asia has led to heightened concern about the environment as a transmission route for AMR to humans.

Bacterial strains that carry the NDM-1 gene are resistant to many antibiotics and can transmit this gene to other bacteria through a process called horizontal gene infection. NDM-1 has been established in Bangladesh since 2010. Bangladesh's environment is ideal for rapid expansion of AMR-high density populations; Lack of pure drinking water; Weak infrastructure for sanitation; and the availability of cheap antimicrobials from over the counter suppliers. Inadequate control over the determination of antimicrobials in the environment through production and manufacturing processes adds further complexity to the development and spread of resistance in the environment. These problems need to be addressed without delay. To explore the AMR transmission between the outdoor environment and human and animal hosts, we consider poultry as one of the main drivers of AMR selection through AMR bacterial fecal shedding. The rapid expansion of commercial poultry farms in the Perry-urban area carries risks due to uncontrolled use of antibiotics for feed and treatment and lack of any control over waste disposal. Dhaka Fresh food and poultry markets are the largest commercial poultry outlets in Dhaka city, where a large number of birds are slaughtered and processed on a daily basis without any controlled waste disposal system. Fecal waste produced on commercial farms and city markets is mostly dumped into the environment through direct washing.

6.3. Co-presence of heavy metals and antibiotic resistance in microorganisms due to arsenic contamination - Discharge of arsenic through drinking water is a serious public health problem in Bangladesh. This concern is further strengthened by the fact that arsenic release may contribute to the improvement of AMR in bacteria. Recent evidence from animal models and wild bacteria in the ancient environment has shown that extreme environmental microorganisms that come in contact with heavy metals, including arsenic, form a defense against these metals that simultaneously provide the microorganisms with resistance to antibiotics. Furthermore, exposure to high environmental concentrations of heavy metals has been shown to be sufficient to maintain multidrug resistance genes in clinically relevant opportunistic pathogens such as $E$. coli and $K$. pneumoniae. We want to find out if this happens in humans in areas where people are repeatedly exposed to arsenic through contaminated water. We have ideal conditions to do this study as we have areas with high level of ground water arsenic contamination and people have a regular exposure to this water. On the other hand, the rate of infectious diseases is very high and infection with MDR organ-isms is an overarching problem in the community.

\subsection{Pathways of antibiotic use among people and} animals in Bangladesh - Presence of over-the-counter antibiotics for human and animal health and lack of quality control among healthcare providers (including an array of unregulated or under-regulated private suppliers), significant developments in antibiotic resistance in Bangladesh and the vast Southeast Asia region. The development of human antibiotic resistance is closely linked to the use of antibiotics in animals. Understanding the behavior and priorities of these healthcare providers (HCPs) and the needs of buyers who utilize them are central to the development of effective AMR strategies. There are many reasons why people seek antibiotic treatment and the beliefs and meanings associated with antibiotics affect the way they are used. In rural areas, the same antibiotic 
suppliers are used for livestock and poultry as well as for humans. We are about to launch a new study to identify ways to promote behavioral change among practitioners, livestock owners, and people/patients using antibiotics. Using a variety of approaches we will try to explore people's beliefs and exposure to identify the role of antibiotics in curing ill health and ways to improve prescribed methods while maintainning their access. We will examine the holistic-calli home use of antibiotics, including the use of animals and humans, through a one-health approach.

\section{Prevent antibiotic resistance}

7.1. Physicians, nurses, veterinarians and other health workers - Do not prescribe or dispense antibiotics unless they are really necessary and you should test any antibiotics for your human patient or the animal you are treating and you have done everything you can to make sure. Today it is estimated that in half of all cases antibiotics are prescribed for viral conditions, where they are of no good use. Clean your hands, instruments and environment and you can do more to prevent infection by using vaccines where appropriate.

7.2. People using health care - Take antibiotics only if prescribed by a reputable health professional, but don't be afraid to ask if you think you really need them. If you are taking any antibiotics, always complete the full prescription even if you feel better, because you are stopping the treatment early promotes the growth of drug-resistant bacteria.

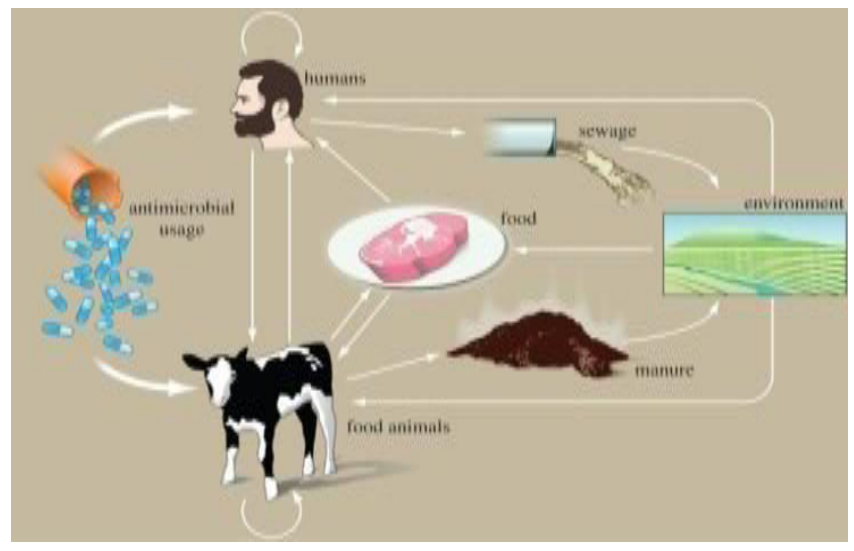

Fig 9: Mechanism of prevention of antibiotic resistance.

7.3. Farmers and others in the agriculture field Ensure that antibiotics given to animals are utilized UniversePG I www.universepg.com only to prevent or treat pathogenic diseases and under the supervision of a veterinarian. Antibiotic Abuse Life-Tuck, aquaculture and crop antibiotic resistance The environment and the food chain and the main reasons for its spread among humans may reduce antibiotic use due to clean and grooved conditions and animal vaccinations.

7.4. Governments - We need a strong national action plan to tackle antibiotic resistance. Serious measures include improving the surveillance of antibioticresistant infected ions, controlling the proper use of standard medicines and educating them about the risks of overuse.

7.5. Development Organizations - Compared with populations in industrialized nations, people in lowincome countries are not getting fair access to antibiotics. Guidance is needed to ensure that the necessary antibiotics are affordable, reach out to the people who really need them and use them responsibly, when donors seek help to strengthen their health systems.

7.6. Consequences of AMR - Infections caused by antimicrobial-resistant bacteria often fail to respond to standard drug therapy. This leads to chronic illness and long-term infectivity. When contaminants are resistant to 'front-line' antimicrobials, second-third or third-line drugs are dispensed, which are generally more costly and sometimes more dangerous. For example, the combination of drugs needed to treat multidrug-resistant forms of tuberculosis (MDR-TB) are more than 100 times more expensive than the combination of front-line drugs used to treat nonresistant forms of TB. Longer hospitalization periods for patients also increase the financial burden to healthcare authorities. Drug-resistant infections also compromise the success of treatments such as organ transplantation, cancer chemo-therapy and major surgery.

7.7. Industry - The industry needs to move faster and more aggressively to research and develop new antibiotics, but we also need to apply new ways to stimulate research and development. Many antibiotics from the late 1980s discuss "discovery void". We are currently in a race between drug development and bacterial evolution. Encouragement to develop new antibiotics can help. There are some enthusiastic 
trends. For example, the European Union, the United Kingdom, the United States, and Canada have advanced projects to fill this knowledge gap by funding scientists directly and for clinical research; Working with pharmaceutical companies; and offering monetary prizes for new diagnostics needed to use antibiotics more responsibly. But it is clear that a new, more modern method is needed. The WHO and Medicines for Neglected Diseases are working to create a global antibiotic research and development facility for enterprises that will work closely with the pharmaceutical industry, universities, civil society and health authorities worldwide.

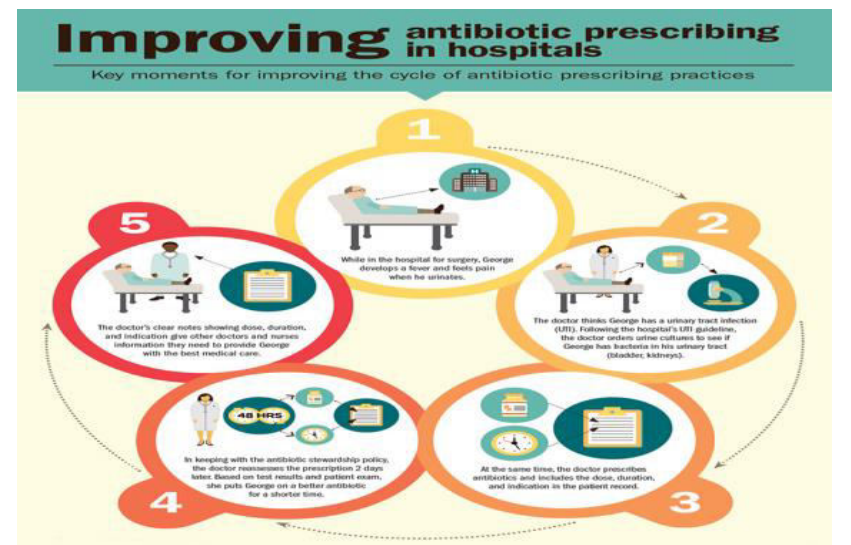

Fig 10: Mechanism of improving antibiotic prescribing.

The partnership will also ensure that new drugs are affordable for all and embed the need for conservation of new antibiotics in the development process. Without a novel system for using antibiotics, we will repeat failed historic efforts to capitalize on one of the greatest scientific discoveries the world has ever seen. Time is running out.

7.8. Improving antibiotic prescribing - Prescribing antibiotics when they are not needed or prescribing the wrong antibiotic in outpatient settings such as doctors' offices is common. In some cases, doctors may not order laboratory tests to confirm whether a bacterial infection is occurring, and therefore antibiotics may be prescribed unnecessarily. In other cases, patients demand treatment for conditions like winter when antibiotics are not needed and do not help. Similarly, healthcare providers may be interested in meeting a patient's expectations of antibiotic press-cropping. The CDC Gate conducts smart programs, conducts a national campaign to improve the prescription and use UniversePG I www.universepg.com of antibiotics in both outpatient and inpatient areas, and supports a variety of state-based programs designed in a national effort. CDC provides messages and resources to local public health authorities to improve antibiotic use in outpatient settings and is now working with a variety of partners to identify new approaches for improving antibiotic use.

7.9. Limiting and interrupting the spread of antibiotic-resistant infections in the community - Preventing the spread of infection in the community is a significant challenge, and many prevention interventions are used, depending on the type of infection and the route of transmission.

7.9.1. Contact tracing - A prevention strategy that has proven successful is tracking cases and tracing contacts. This process is used to ensure that all persons requiring an intervention such as treatment, prophylaxis, or temporary isolation from the general public are identified and managed appropriately. This approach is resource intensive, but it has successfully limited transmission of infections including tuberculosis, gonorrhea, and meningococcus.

7.9.2. Vaccination - There are few vaccines for antibiotic-resistant bacteria, but the $S$. pneumonia vaccine has proven that an effective vaccine can reduce antibiotic resistance rates. The vaccine targets certain types of the bacteria, even if it is a resistant type, and reduces the overall number of infections, including those that are caused by resistant strains. The first version of the vaccine was introduced in 2000 and reduces the frequency of antibiotic-resistant infected ions, but it does not protect against a specific strain called S. pneumoniae serotype 19A. This strain gradually becomes resistant to antibiotics and causes further infections because the vaccine does not provide protection. A new version of the vaccine approved for use in 2010 protects against serotype 19A. As a result, the rate of resistant pneumococcal infection is declining.

7.9.3. Treatment guidelines - The spread of antibiotic resistance can be prevented if infections are effectively treated before the pathogen is spread to others. For some infections, laboratory tests for treatment management are not readily available or the rotation time is slow or incomplete. It is used in CDC 
monitors resistance trends in $N$. gonorrhoeae and $M$. tuberculosis and focuses treatment plans to limit the expression of these diseases and expand of bacteria.

7.9.4. Promotion of safe sex - Increasing the prevalence of drug-resistant Neisseria gonorrhea is a unique challenge. To prevent the transmission of this virus, the CDC promotes safe sexual practices such as exclusion, intercourse, and proper and consistent condom use.

7.10. Preventing infections - Efforts to prevent food borne and other enteric infections help to reduce both antibiotic-resistant infections and antibiotic-susceptible infections. They help prevent these infections include: Estimating how much food borne illness occurs, Monitoring trends in food borne infections, Attributing illnesses to specific foods and settings, Tracking and responding to changes in resistance, Determining the sources of antibiotic-resistant enteric infections, Educating consumers and food workers about safe food handling practices, Identifying and educating groups at high risk for infection, Promoting proper hand washing, Strengthening the capacity of state and local health departments to detect, respond to, and report food borne infections, Developing better diagnostic tools to rapidly and accurately find sources of contamination, and Providing recommendations for travelers on safe food and clean water (WHO, 2015; CDC, 2020).

\section{CONCLUSION:}

AMR is not just a problem in medical science that is struck by nature; Human actions and distorted motivations play a major role in its development. The misuse of antibiotics in the medical, veterinary and agricultural sectors, including inappropriate prescription of antibiotics, their overuse in the livestock sector and inadequate hygiene in hospitals, all contribute to AMR growth. Global trade and travel are also accelerating. At the same time, the development pipeline of new antibiotics has slowed down, largely due to inadequate stimulation, leading to the developpment of new drugs for microorganisms. AMR carries a significant burden on the healthcare system and the national budget. The discovery of antibiotics meant that previously the process of curing deadly infections the treatment of gonorrhea and tuberculosis. For these UniversePG I www.universepg.com infections, healthcare providers rely on treatment guidelines for proper management of the infection and surgical procedures had been made safer, allowing for the development of modern medicine. With the rise of AMR we are now moving towards a 'post-antibiotic era' where common infections can once again be fatal and Patients with resistant infections.

\section{ACKNOWLEDGEMENT:}

The authors would like to thank the authority of the Dept. of Pharmacy, ASA University Bangladesh, Dhaka for the valuable suggestions in writing this article and also all kind of support.

\section{CONFLICT OF INTERESTS:}

The authors declare no conflict of interest.

\section{REFERENCES:}

1. Alam S. A. M. F., Alam M. G., Maitra P., Uddin ME., Ahammed T. (2017). Synthesis of benzimidazole derivatives containing schiff base exhibiting antimicrobial activities. International J. of Research Studies in Biosciences, 5(7), 18-24. https://doi.org/0.20431/2349-0365.0507003

2. Babic M., Hujer A. M., and Bonomo R. A. (2006). "What's new in antibiotic resistance? Focus on beta-lactamases," Drug Resistance Updates, 9(3), pp. 142-156.

https://pubmed.ncbi.nlm.nih.gov/16899402/

3. Bonnet R. (2004). "Growing group of extended spectrum $\beta$-lactamases: The CTX-M Enzymes," Antimicrobial Agents and Chemotherapy, 48(1), pp. 1-14.

https://doi.org/10.1128/AAC.48.1.1-14.2004

4. Bush K. and Jacoby G. A. (2010). "Updated functional classification of $\beta$-lactamases," Antimicrobial Agents and Chemotherapy, 54(3), pp. 969-976. https://doi.org/10.1128/AAC.01009-09

5. Carmeli Y, Eliopoulos G, Mozaffari E, Samore M. (2002). Health and economic outcomes of vancomycin resistan enterococci, Arch Intern Med. 162, pp. 2223-8. https://doi.org/10.1001/archinte.162.19.2223

6. Carmeli Y, Troillet N, Karchmer AW, Samore MH. (1999). Health and economic outcomes 
of antibiotic resistance in Pseudomonas aeruginosa, Arch Intern Med, 159, 1127-32.

7. CDC, (2020). Antibiotic/ Antimicrobial Resistance (AR/MR). Available at https://www.cdc.gov/drugresistance/

8. Del Pozo J. L. and Patel R. (2007). "The challenge of treating biofilm-associated bacterial infections," Clinical Pharmacology and Therapeutics, 82(2), pp. 204-209. https://pubmed.ncbi.nlm.nih.gov/17538551/

9. Džidić S., Šušković J., and Kos B. (2008). "Antibiotic resistance mechanisms in bacteria: biochemical and genetic aspects," Food Technology and Biotechnology, 46(1), 11-21.

10. Ekhlas Uddin Md., Pulak Maitra, Hossain Md. Faruquee, Firoz Alam Md., (2014). Isolation and characterization of proteases enzyme from locally isolated Bacillus sp., American Journal of Life Sciences. 2(6), 338-344. https://doi.org/10.11648/j.ajls.20140206.12

11. Giedraitienè A., Vitkauskienè A., Naginienè R., and Pavilonis A. (2011). "Antibiotic resistance mechanisms of clinically important bacteria," Medicina, 47(3), pp. 137-146. https://pubmed.ncbi.nlm.nih.gov/21822035/

12. Happy A. H., Alam M. G., Mahmud S., Uddin M. E. (2018). Isolation, identification \& characterization of gram-negative bacteria from popular street food (Chotpoti) at Savar area, Dhaka, Bangladesh, Open Access Library J. 5, e4986. https://doi.org/10.4236/oalib.1104986

13. Høiby N., Bjarnsholt T., Givskov M., Molin S., and Ciofu O. (2010). "Antibiotic resistance of bacterial biofilms," International J. of Antimicrobial Agents, 35(4), 322-332. https://pubmed.ncbi.nlm.nih.gov/20149602/

14. Islam R., Parvin A., Imran M. A. S., Sarker R. K., Uddin M. E., Alam M. S., and Abedin M. Z. (2020). Assessment of the effects of genetically modified (GM) foods: a brief study on health and environmental concerns, J. of Material and Environmental Sciences, 11(10): 1676-1688,

https://www.jmaterenvironsci.com/Document/vo 111/vol11_N10/JMES-2020-11144-Islam.pdf
15. Jacoby G. A. and Munoz-Price L. S. (2005). "The new beta-lactamases," The New England journal of medicine, 352(4), pp. 380-391.

16. Jacoby G. A. (2009). "AmpC $\beta$-lactamases," Clinical Microbiol. Reviews, 22(1), 161-182. https://cmr.asm.org/content/22/1/161

17. Klevens R. M., Edwards J. R., Richards C. L. Jr. et al. (2007). "Estimating health careassociated infections and deaths in U.S. Hospitals, 2002," Public Health Reports, 122(2), pp. 160-166.

https://doi.org/10.1177/003335490712200205

18. Kumarasamy K. K., Toleman M. A., Walsh T. R. et al. (2010). "Emergence of a new antibiotic resistance mechanism in India, Pakistan, and the UK: a molecular, biological, and epidemiological study," The Lancet Infectious Diseases, 10(9), pp. 597-602.

19. Laverty G., Gorman S. P., and Gilmore B. F. (2014). "Biomolecular mechanisms of Pseudomonas aeruginosa and E. coli biofilm formation," Pathogens, 3(3), pp. 596-632. https://pubmed.ncbi.nlm.nih.gov/25438014/

20. Li X.-Z., and Nikaido H. (2004). "Effluxmediated drug resistance in bacteria," Drugs, 64(2), pp. 159-204.

https://doi.org/10.2165/11317030-000000000$\underline{00000}$

21. Magill S. S., Edwards J. R., Bamberg W et al. (2014). "Multistate point-prevalence survey of health care-associated infections," The New England Journal of Medicine, 370(13), 11981208. https://doi.org/10.1056/NEJMoa1306801

22. Mondol GC, Alam MG, Rahman S, Hossain MA and Uddin ME. (2018). Prevalence of Antibiotic Resistant Staphylococcus aureus Among Patients who come to Seek Treatment in a Hospital of Bangladesh. Clinical Biotechnology and Microbiology, 2(5), 451- 455. https://scientiaricerca.com/srcbmi/pdf/SRCBMI02-00065.pdf

23. Nikaido H. and Pagès J.-M. (2012). "Broadspecificity efflux pumps and their role in multidrug resistance of Gram-negative bacteria," FEMS Microbiol. Revi., 36(2), 340-363. https://doi.org/10.1111/j.1574-6976.2011.0029 0.x 
24. Olivier Jolliet, (2005). Spread of antibiotic resistance in the environment and in humans, School of Public Health, University of Michigan, USA.

25. Pucci M. J. and Dougherty T. J. (2002). "Direct quantitation of the numbers of individual penicillin-binding proteins per cell in Staphylococcus aureus," J. of Bacteriology, 184(2), pp. 588-591.

https://www.ncbi.nlm.nih.gov/pmc/articles/PMC 139569/

26. Rahman MA, Ahmad T, Mahmud S, Uddin ME, and Ahmed R. (2019). Isolation, identification and antibiotic sensitivity pattern of Salmonella spp. from locally isolated egg samples, Am. J. Pure Appl. Sci., 1(1), 1-11. https://doi.org/10.34104/ajpab.019.019111

27. Rice L. B. (2008). "Federal funding for the study of antimicrobial resistance in nosocomial pathogens: no ESKAPE," Journal of Infectious Diseases, 197(8), pp. 1079-1081. https://doi.org/10.1086/533452

28. Rice L. B. (2010). "Progress and challenges in implementing the research on ESKAPE pathogens," Infection Control and Hospital Epidemiology, 31(1), pp. S7-S10. https://doi.org/10.1086/655995

29. Schweizer H. P. (2003). "Efflux as a mechanism of resistance to antimicrobials in Pseudomonas aeruginosa and related bacteria: unanswered questions," Genetics and Molecular Research, 2(1), pp. 48-62. https://pubmed.ncbi.nlm.nih.gov/12917802/

30. Shahen MZ, Mahmud S, Uddin ME and Alam MS. (2019). Effect of antibiotic susceptibility and inhibitory activity for the control of growth and survival of microorganisms of extracts of Calendula officinalis, Eur. J. Med. Health Sci. 1(1), 1-9. https://doi.org/10.34104/ejmhs.0190109

31. Sharma G., Rao S., Bansal A., Dang S., Gupta S., and Gabrani R. (2014). "Pseudomonas aeruginosa biofilm: potential therapeutic targets," Biologicals, 42(1), pp. 1-7. https://pubmed.ncbi.nlm.nih.gov/24309094/

32. Sun J., Deng Z., and Yan A. (2014). "Bacterial multidrug efflux pumps: mecha-nisms, physiology and pharmacological exploitations," Biochemical and Biophysical Research Communications, 453(2), 254-267. https://doi.org/10.1016/j.bbrc.2014.05.090

33. Tang S. S., Apisarnthanarak A., and Hsu L. Y. (2014). "Mechanisms of $\beta$-lactam antimicrobial resistance and epidemiology of major community and healthcare-associated multidrug-resistant bacteria," Advanced Drug Delivery Reviews, 78, pp. 3-13.

https://pubmed.ncbi.nlm.nih.gov/25134490/

34. Thomson J. M. and Bonomo R. A. (2005) "The threat of antibiotic resistance in Gramnegative pathogenic bacteria: $\beta$-lactams in peril!," Current Opinion in Microbiology, 8(5), pp. 518-524. https://doi.org/10.1016/j.mib.2005.08.014

35. Uddin M. E., Ahmad T., Ray S. K., and Ahammed T. (2017). Thermotolerant extracellular proteases produced by B. subtilis isolated from local soil that representing industrial applications. J. of Pure and Applied Microbiol. 11(2), 733-741. https://doi.org/10.22207/JPAM.11.2.12

36. US Congress, (1995). Impacts of antibioticresistant bacteria: thanks to penicillin-he will come home!, 1995 Washington, DC. Office of Technology Assessment, Congress.

37. US FDA, (2004). Animation of Antimicrobial Resistance (text version), Content current as of: 04/30/2020. Available at -

https://www.fda.gov/animal-veterinary/antimi crobial-resistance/animation-antimicrobial-resis tance-text-version

38. Vaez H., Faghri J., Isfahani B. N et al. (2014). "Efflux pump regulatory genes mutations in multidrug resistance Pseudomonas aeruginosa isolated from wound infections in Isfahan hospitals," Adv. Biomedical Research, 3(1), 117. https://doi.org/10.4103/2277-9175.133183

39. WHO, (2015). How to stop antibiotic resistance? Here's a WHO prescription. 20 November 2015. Available at https://www.who.int/mediacentre/commentaries/ stop-antibiotic-resistance/en/ 
40. WHO, (2017). WHO publishes list of bacteria for which new antibiotics are urgently needed; 27 February 2017, News release GENEVA. https://www.who.int/en/news-room/detail/2702-2017-who-publishes-list-of-bacteria-forwhich-new-antibiotics-are-urgently-n

41. WHO, (2020). Antibiotic resistance, 31 July 2020. Available at https://www.who.int/en/news-room/fact-sheets/ detail/antibiotic-resistance

42. Wilson D. N. (2014). "Ribosome-targeting antibiotics and mechanisms of bacterial resistance," Nature Reviews Microbiol., 12(1), 35-48. https://doi.org/10.1038/nrmicro3155

43. Wright G. D. (2005). "Bacterial resistance to antibiotics: enzymatic degradation and modification," Advanced Drug Delivery Reviews, 57(10), pp. 1451-1470. https://pubmed.ncbi.nlm.nih.gov/15950313/

44. Yong D., Toleman M. A., Giske C. G et al. (2009). "Characterization of a new metallo- $\beta$ lactamase gene, bla NDM-1, and a novel erythromycin esterase gene carried on a unique genetic structure in $K$. pneumoniae sequence type 14 from India," Antimicrobial Agents and Chemotherapy, 53(12), pp. 50465054. https://doi.org/10.1128/AAC.00774-09

45. Zhao W. H., and $\mathrm{Hu}$ Z. Q. (2013). "Epidemiology and genetics of CTX-M extended-spectrum $\beta$-lactamases in Gramnegative bacteria," Critical Reviews in Microbiology, 39(1), pp. 79-101. https://doi.org/10.3109/1040841X.2012.691460

Citation: Rahamn MA, Rahman B, Ahammed T, and Sharif IH. (2020). Exploration of the antimicrobial resistance and their remedy in living world: a brief study, Eur. J. Med. Health Sci., 2(5), 86-100. https://doi.org/10.34104/ejmhs.020.0860100 (c) क 